Voix et Images

voixetimages

\title{
Interprétation de quelques formes des discours de Gabrielle Roy
}

\section{René Juery}

Volume 6, numéro 2, hiver 1981

Jean-Claude Germain

URI : https://id.erudit.org/iderudit/200270ar

DOI : https://doi.org/10.7202/200270ar

Aller au sommaire du numéro

Éditeur(s)

Les Presses de l'Université du Québec

\section{ISSN}

0318-9201 (imprimé)

1705-933X (numérique)

Découvrir la revue

Citer cet article

Juery, R. (1981). Interprétation de quelques formes des discours de Gabrielle Roy. Voix et Images, 6(2), 293-317. https://doi.org/10.7202/200270ar d'utilisation que vous pouvez consulter en ligne.

https://apropos.erudit.org/fr/usagers/politique-dutilisation/ 


\title{
Interprétation de quelques formes des discours de Gabrielle Roy
}

\author{
par René Juery
}

Les études du récit initlées par Propp et Sourlau ont permis, et ce n'est pas le moindre de leur mérite, de poser les fondallons d'une grammaire transphrastique et d'ouvrir la voie ce que l'on eppelle perfols une macrostylistique. Il est des lors possible de concevoir, du point de vue de la réception, l'effet de sens comme étent réparti dans un syntagme de dimension égale ou supérieure a la phrase et, du côté de la production du sens, d'átudier les figures d'une dimension correspondante. La faveur. dont jouit actuellement lintertexlualité ne semble pas etrangere a cette pouss ée vigoureuse vers l'étude de la dynamique des macro-structures.

Caduque l'étude de symbolisme lexical? Nous ne le pensons pas, mais nous ferons place ici à un symbolisme élargi tant dans la délinition de ses propriétés que dans sa manilestation linguistique. Nous dirons qu'il y a sens indirect dès que le rapport entre deux unités linguistiques paraît incongru au lecteur, lequel utilise alors sa compétence linguistique pour modifier le sens premier (direct) et rendre ainsi à la choine syntagmatique toule sa pertinence. Nous dirons que le lecteur a interprété le discours.

L'objet de nos interprétations est une couvre de Gabrielle Roy qui semble avoir été négligée par la critique et par les lecteurs. Elle a pour titre Cet été qui chantait'. Nous commencerons par l'étude des quátre textes les plus courts du recueil.

II nous semble, en ellet, que l'analyse textuelle trouve son avantage a rapprocher le plus possible le texte de dépent du (ou des) texte(s) d'arrivée. Une fois détaillé le fonctionnement des types de sens indirecıs caractéristiques de l'ceivre, nous entreprendrons une lecture plus rapide de chacune des autres pieces selon l'ordre dans lequel elles apparaissent dans le recueil. Notre intention est d'utiliser le présent travail comme base rélérentielle pour l'étude d'onuvres plus volumineuses ei moins fragmentables du mene auteur.

Enfiu, un dernier mol sur la proceddurb. Nous analyserons les sens indirects lans la coulóe méme de leurs découvertes, c'est-h-dire dans le vácu de le lecture. Nous utiliserons les ligures rhétoriques comme des modèles 
provisoires ouverts à des emplois (et à des effets) nouveaux. Nous témoignons ainsi de notre intention de suivre au plus près une démarche pragmatique.

\section{*LES VACHES D'AIMÉ.}

Je coupe à travers champs pour me rendre chez mon voisin. Les vaches d'Aimé à mon passage s'arrêtent de brouter. Elle lèvent la tête, me regardent, me suivent des yeux comme pour bien me situer. Je ne sais pourquai on les dit bêtes. Leur regard, alors qu'elles m'examinent à fond et notent des détails indique clairement une sorte de réflexion.

«Ah bon! C'est la dame du chalet, qui s'en va encore chez notre maître. Celle qui vient de la ville. Qui passe lété par ici."

Et de se remettre a brouter tranquillement. La preuve que tout doit se dérouler à peu près de cețte facon dans leur esprit, c'est que si je repasse dix minutes plus tard elles me regardent un bref moment mais sans prendre la peine de cesser de brouter et de lever la tête comme si elles concluaient: “C'est la même que tantót. .

Mais sije ne repasse que deux ou trois heures plus tard, alors le mandge recommence: les vaches cessent de brouter; elles me suivent des reux, m'examinent avec une curiosité renouvelée. Et puis entin: «Ah! mais oui! C'est la dame de ce matin. Celle du chalet. Celle qui vient de la ville. Celle qui va chez notre maître cent fois par jour."

C'est comme si elles manquaient de mémoire plutôt que de bon sens.

Ou comme si elles étaient tout aussi distraites que certaines gens qui ne «replacent» pas leurs connaissances.

Ma théorie ne tient plus. Airepassé deux fois aujourd'huipar les champs à une heure d'intervalle. La deuxieme comme la premiere fois les vaches ont cessé de brouter. Mont longuement examinée. Dévisagée pour ainsi dire. Ont manifesté la même curiosité que deux heures auparavant. Que la veille.

J'en ai parlé à Aimé.

- Me reconnaissent-elles, pensez-vous? Reconnaissent-elles les gens?

- Ah oui!

- Alors?

- Alors ! Elles me trouvent donc si étonnante?

Aimé, poliment:

- Ca se peut... des fois!

Les deux premières phrases portent les marques nombreuses d'une énonciation qui instaure le sujet parlant non seulement comme le narrateur 
de son propre discours mais aussi en tant qu'acteur tantôt sujet tantôt objet de son énoncé. Nous l'appellerons Narrateur-Témoin et le désignerons désormais par NT. La référence au "voisin Aimén accentue le caractère descriptif, littéral de ce discours.

Cependant, en lisant la troisième phrase, le lecteur est mis en alerte par l'indice d'un sens indirect. II s'agit de la conjonction acommen qui associe de façon insolite la description d'une série d'actions d'un acteur-sujet de l'énoncé. les vaches, à la finalité de leurs actions. Nous citons : «Elles (les vaches) lévent la tête. ... comme pour bien me situer * (35). Autrement dit, deux termes dont la contiguïté ne lait pas problème sont séparés par une conjonction qui, d'ordinaire, introduit une équivalence partielle entre deux éléments linguistiques.

La réduction de chacun des termes à une forme substantivale, par exemple à «regard des vaches " et «intentionnalité de ce regard * fait mieux apparaitre la mise en relation d'équivalence partielle entre, au plan sémantique. deux contenus, l'un appartenant au domaine du sensible, l'autre à celui de l'intelligible et, au plan grammatical, entre une action décrite et sa modalité.

En d'autres mots, la mise en équivalence cherche a laire croire au lecteur que le deuxième terme est partiellement contenu dans le premier. Mais, en fin de compte, la tinalité des gestes des animaux est rejetée dans l'irréel ou dans l'hypothétique, par opposition aux gestes eux-mémes qui appartiennent au singulier, à ce qui est arrivé, à l'histoire. Ainsi l'hésitation du producteur du sens asserter un phénomène étrange est transmise au récepteur, tout au moins pendant un instant.

Mais la suite immédiate du texte transformel'incident en une illustration de l'opinion toute faite du NT sur l'espèce bovine. L'énoncé sentencieux an les dit bêtes * est couvert par l'énonciation a je ne sais pourquoi * qui suggère plus une indignation du NT devant l'opinion commune qu'un aveu d'ignorance de sa part. C'est ce que confirme d'autres marques de l'énonciation, par exemple "clairement o dans la phrase suivante qui est appuyée par une citation directe du discours prêté aux vaches: «ah bon, c'est la dame du chalet...*. Toutefois la distribution définitive du caractère «intelligence * a l'espèce vache reste à nouveau en suspens lorsque le Narrateur révèle que la citation du discours direct des vaches était en réalité une construction de son esprit (cf a tout doit se dérouler à peu pres de cette façon.m).

Par la suite, l'énonciation ne cesse d'accentuer le caractère hypothétique de la distribution proposée en utilisant en particulier la même conjonction, mais en lui ajoutant cette fois une marque plus directe de I'hypothese, la conjonction *si *.

Nous allons nous attarder sur les deux dernières manifestations de l'éventualité qui caractérise l'énonciation du Narrateur dans son entreprise de description.

On les rencontre l'une à la suite de l'autre. Nous citons: „C'est comme si elles manquaient de mémoire plutôt que de bon sens. Ou comme si elles 
étaient tout aussi distraites que certaines gens. - La distriburıon suggérée dans la première proposition est le bon sens par élimination d'une autre qualité. la mémoire, élimination elle-mème indécidable, non assertée vigoureusement (cf aplutôt que•). La deuxième proposıtion reconnaîtrait une autre qualité humaine. la distraction.

Mais ce qui retient notre attention, c'est que ces deux propositions sont reliées par une conjonction de coordination cou $=$ qui les transforment en termes d'une alternative qui donne brusquement à la démarche du Narrateur une plus grande rigueur. En effet, chaque terme est exclusif l'un de lautre au sein d'un ensemble homogène qui peut ètre reconnu comme une typologie de l'espèce :vaches *. On aurait alors des classes d'individus ayant des sèmes communs (bon sens, ...) mais aussi de traits distinctifs permettant leur regroupement en sous-especes comme les Distraites, les Rêveuses, les Tronteuses. les Amnésiques,...

On passerait ainsi de la description matérielle de l'univers sémantique à une approche plus conceptuelle. Mais celle-ci s'effectue dans le cadre d'une description aberrante du réel qui postule le sensible comme directement intelligible. On peut se demander quel est l'acquis d'une telle analyse des sens indirects principaux par rapport à une lecture plus littérale. La procédure la meilleure pour en rendre compte. parait être de mettre en présence le texte d’arrivée d'une lecture compréhensive et celui d'une lecture interprétative. Telle sera notre démarche. Nous sommes bien conscients que, d'une part, les deux lectures sont faites par la mème personne et que, d'autre part, la frontière entre interprétation et compréhension ne peut être rigoureusement délimitée.

Voici donc le texte d'arrivée edénotatif \& que nous proposons: Le Narrateur observé régulièrement et de façon insistante par des vaches retourne son propre regard vers les animaux. II interprète leur conduite dans une perspective anthropomorphique. Le dérèglement de ce comportement constitue pour le NT un événement déroutant qui l'amène à demander assistance à la personne compétente. Aimé. le voisin possesseur des vaches. Celui-ci confirme la justesse des premières observation ce qui induit le NT a ajouter aux facultés intellectuelles des animaux, dès lors reconnues, celles d'éprouver des émotions, ou d'avoir des sentiments esthétiques (ct - Elles me trouvent donc si étonnante p. 36). Mais. d'une part. l'attribution d'une telle qualité est suspendue a une réponse, laquelle, d’autre part. est évasive.

Passons maintenant à une lecture plus interprétative. La réponse de normand d'Aimé correspond justement à la modalité gnoséologique du doute qui semble gouverner l'énonciation du NT. Elle enlève aux phrases affirmatives toute leur force assertive. Tout le travail de décomposition et de recomposition de l'univers sémantique de l'être evache est rongé par le non engagement du sujet connaissant, par son incertitude quant au recoupement possible des univers animal et humain. Or, tous les éléments de l'énonciation analysés traduisent le caractère sérieux, sinon rigoureux, de la démarche entreprise.

Aussi, le point culminant du discours du NT. la - pointe = selon un langage poétique (cf «Ca se peut... des fois!* p. 36) peut être interprétée comme un 
euphémisme, une litote, ou comme une remarque ironique faite par le professionnel Aimé à l'amateur. De telles figures, voisines d'ailleurs, sont probablement construites de facon consciente par le NT (ct apoliment ") pour camoufler le sentiment final de sa supériorité sur la créature "vache". Autrement dit, la lorme enjouée du dialogue avec Aimé n'arrive pas à cacher l'importance de l'entreprise de connaissance qui est en définitive, celle de soi.

L'interprétation des sens indirects semble bien ouvrir la voie à des lectures diverses qui ne sont pas forcément exclusives l'une de l'autre. Elle doit également préparer à l'étude diachronique de la production du sens chez le même auteur.

\section{"LA TROTTEUSE"}

Aujourd hui les trois vaches d'Aime se tiennemt les pattes dans l'eau du minuscule dtang pres de chez moi. Elles broutent les Heurs qui entourent ce point deau, surtout des lysimaques en cette saison. De temps autre, lune se penche, goute l'eau tiédie par le soleil, la deguste plutót qu'elle ne la boit. La Trotteuse porte au cou la clochette qu'Aime a couru d'une ripouste lui acheter a Baie-Saint-Paul, afin de la reperer lorsqu'il l'appelle el qu'elle reste des heures cachée dans les aulnes à refuser de se montrer.

\section{bougraise.}

- Je m'en vas toujours bien savoir au moins où elle se tient, la

Depuis tinte la clochette au moindre mouvement de la bougraise et toujours on sait où elle est. Du reste l'envie de se cacher semble lui avoir passé maintenant que le grelot à son cou annonce sa présence où quelle aille.

Le timbre en est mélodieux, doux et charmant à entendre.

Si c'est le grand calme, il n'y a pas d'autre bruit et on en est tout réjoui. s'il fait du vent, le son émerge a peine du brassage des feuilles. Quelquefois il nous parvient de si loin quion a l'impression que c'est d'un autre temps, d'un autre monde.

Mais aujourd'hui les trois vaches se som rapprocheses les unes des autres. Les pattes dans l'eau. elles revent de longs momemts, les yeux a peine levés de terre. lixés devam elles sans expression, telles bien des gens que je connais lorsqu'ils poursuivent sans trop le savoir une idée vague.

Car, depuis que la Troffeuse seme de la musique autour d'elle, curieusement les deux autres vaches ne la quittent plus. Ainsi, quand Aimé repere la Trotteuse, du méme coup il repere les autres.

Il y a un instant les trois vaches se sont approchées de ma barriere. comme mues par la curiosité soudnine de savoir ce que je deviens. Si seulement elles apprenaient que i écris sur elles!

La clochelle a tinté fortement, tout pres. El pourquoi cela a-t-ilbrusquement éveillé en moi le souvenir - que je crovais mort - du temps où, enfamt. 
lorsque jarrivais pour les vacances d'été chez mes ancles sur leurs fermes au Manitoba, j'étais accueillie dès en descendant du train par le drelin d"une cloche à main qu'agitait sur le seuil l'hötelier venu annoncer un repas chaud et tout prêt... et que i"en étais rendue heureuse mystérieusement comme si nous étions conviés ensemble. les inconnus sur le quai, à une sorte de repas de fête. d'amitié.

Parce que Aimé, fâché contre sa vache, lui a passé au cou une clochette, voici que m'est redonnée cette curieuse joie de ma vie dont je ne sais toujours pas au juste de quoi elle est faite et pourquoi elle m'enchante encore.

On retrouve dans ce texte, le septième du recueil, les mèmes acteurs: les vaches et le décrypteur de leurs comportements, le NT. Comme précédemment, il y a réciprocité entre les fonctions syntaxiques de ces acteurs: ils sont à tour de rôle sujet et objet.

Le récit s'articule autour du son produit par le grelot d'une vache. Une première partie fait l'historique de ce grelot et raconte la transformation de l'animal qui le parte. Un deuxième mouvement révèle la propre transformation du NT à la suite d'un tintement plus fort.

Ainsi, la première partie du texte explique que les vaches restent en contact visuel et auditif avec le NT et avec le fermier depuis que celui-ci a attaché un grelot à la Trotteuse pour contrôler son humeur folâtre. Or, curieusement, le grelot est devenu inutile puisqu'elle ne se cache plus depuis qu'elle le porte. Plus exactement. la fonction référentielle du tintement est devenue caduque, comme le précise la phrase suivante: «Du reste, l'envie de se cacher semble lui avoir passé maintenant que le grelot à son cou annonce sa présence, partout où qu'elle aille. * (p. 59)

Qu'est-ce à dire, sinon que la Trotteuse était motivée lorsquielle se rendait invisible et que, maintenant, elle trouve inutile de participer à un jeu dans lequel elle serait toujours la perdante. Comme le suggère notre emploi du mot ajeu», le comportement premier de la Trotteuse renvoyait à un sens indirect, voulait témoigner de quelque chose, disons: «je suis une solitaire* ou bien, «Qu'on me laisse tranquille», ou bien encore. «J'aime que l'on s'intéresse à moi: cherchez-moi. ou enfin «je suis timide, pudique ».

Le grelot est pour la Trotteuse le symbole de sa soumission a lthomme. Pour le fermier, il reste un signal sonore car il ne «voit > pas le changement survenu chez la vache; il ninterprète pas sa transformation de nomade en sédentaire, de folātre en apathique.

Mais l'intuition qui manque à Aimé se retrouve chez le NT qui reconnaît implicitement à l'animal trois qualités éminemment humaines: la motivation. la répression des tendances, la conscience des efforts inutiles. Certes, le NT n'asserte pas directement une telle sagesse (cf . ... l'envie de se cacher semble lui avoir passé.... motivation refrénée dans l'actuel du récit.

Par contre, tout convie à voir dans le discours la représentation d'un conflit ou d'un jeu cruel entre deux êtres que le NT considère comme étant 
de la même espèce, mais dont l'un possède une autorité exorbitante. Enumé. rons ces indices sémantiques: l'utilisation du verbe "se cacher non filtré par une quelconque modalité, l'obstination première de l'animal à se rendre invisible et celle du fermier à la découvrir, la course précipitée de ce dernier au village, comme pour répondre à un défi (cf "la ripouste *) l'injure à l'adversaire (ala bougraise $*$ ).

Un tel schéma narratif rappelle les efforts de l'enfant pour s'émanciper des contraintes paternelles ou familiales. ou bien les luttes intestines entre pseudo-magiciens pour le pouvoir supréme, autant de formes engageant irrésistiblement sur la voie d'une interprétation psychanalytique. On connait par exemple le jeu d'occultation d'un objet par un enfant qui, selon les observations de Freud distinguait chaque phase de l'alternance par l'un des vocables «Fort» ou «Dan. On sait également que Lacan a vu dans un tel jeu l'origine du langage en même temps qu'une demande d'amour.

Mais nous nous proposons simplement d'évoquer ici trois migrations intertextuelles du motif.

Ce sere tout d'abord les jeux du NT at de sa scour Alicia dans les champs de maïs ou dans le petit bois de chêne proche de la maison natale dans "Alicia” (Rue Deschambauh p. 149 et 153). Nous rapprocherons également "La Trotteuse» des «Déserteuses» (dans Rue Deschambault) qui raconte comment la mère du NT, poussée par un besoin soudain irrépressible de libération, l'avait entrainée, alors qu'elle était toute petite, dans une longue fugue. Le mari et pere, non averti de ce départ, les avait cherchées en vain et avait rameuté tout le reste de la famille. (On remarquera au passage la paronomase *trotteuse * déserteuse $*$ ). Enfin, nous citerons l'un des personnages principaux de Bonheur d'occasion. Rose Anna, parce que son désir de se cacher parait correspondre à sa honte d'être vue enceinte, déformée (conflit Surmoi-Moi idéal?).

Mais, avons-nous dit, le récit de * La Trotteuse* s'articule autour diun événement-pivat, le tintement soudain plus fort du grelol qui évoque chez le NT un souvenir d'enfance heureux et le transporte d'une joie actuelle. La transformation est décrite dans une longue période qu'une comparaison de type hypothétique fractionne à son tour en deux terme paralleles dont nous allons faire l'analyse.

Le premier terme de l'énonciation (a... j'en étais rendue heureuse mystérieusement x) se décompose en un résultat de la translormation (la joie) et un agent (le drelin représenté par *en*). Quant au terme comparant, il manifeste en outre la puissance de l'agent capable de transformer un simple repas matériel en une communion au sens biblique. Ainsi, le deuxième terme développe l'aspect surnaturel déjà exprimé dans le premier (cf amystérieusement*). Une telle métamorphose ou miracle, suggère que l'hôtelier et son drelin ne sont que les représentạts d'un Destinateur suprême.

Mais, encore une lois, les conjonctions couplées *comme si * atténuent le caractère extraordinaire de la transformation décrite. Tout semble se passer 
comme s'il s'agissait d'une élection, de la réception d'une grâce dont il faudrait cacher l'origine toute païenne ou démoniaque.

Le texte nous apprend que l'épisode rapporté, le ravissement anticipé. se passait dans l'enfance du NT. Le dernier paragraphe asserte la répétition dans l'actuel du récit d'une telle joie, le présentatif a Voici que » lui redonnant la vividité d'une première émergence. II y a donc superposition exacte d'une joie passée, c'est-à-dire d'un souvenir, et d'une joie présente, assurant ainsi à l'être NT une permanence, une continuité sans heurt entre l'enfance et l'àge adulte. La similitude des effets est garantie par l'identité des causes: le grelot de la vache étant équivalent au drelin de l"hôtelier.

Ici, deux interprétations sont possibles. Ou bien il y a confusion entre succession temporelle de deux phénomènes et rapport de cause à effet entre les deux (cf. l'adage *Post hoc ergo propter hoc*). Ou bien il y a parallélisme entre les plans du signifiant et du signifié, celui-là conviant te producteur du sens à celui-ci. Nous voulons parler à nouveau de la figure dite paronomase dont Jakobson fait le cheval de bataille d'une lecture poétique. Elle serait ici constituée par les mots - départ $\alpha$ grelot ". «clochetto (de la vache) et les mots - arrivée «drelin», «cloche» (de l'Hôtelier). Un tel sens indirect pourrait recouvrir un déplacement selon la conception freudienne de la réapparition sous une autre forme de la pulsion refoulée. Les mots en question seraient alors les représentants figurés d'un signifiant (à préciser) monté en position de sujet au sein d'une chaîne symbolique qui resterait à compléter. Nous n'entreprendrons pas une telle recherche puisque la présente étude se veut plus démonstrative de quelques formes des discours de Gabrielle Roy que descriptive de l'univers imaginaire de l'auteur. Un tel parti pris n'est cependant pas refus d'interpréter, comme nous l'avons déjà fait, selon différentes stratégies: soit finaliste (analyse freudienne), opérationnelle (analyse structurale, philologique (intertextualité), génétique (rhétorique).

Aussi nous proposons-nous d'exploiter selon une perspective psychanalytique le parallélisme mis a jour entre les deux parties du texte « La trotteuse . Rappelons que la première racontait l'histoire d'une vache folâtre lou désobéissante, ou...) et la deuxième celle d'un souvenir heureux réactivé. Une telle lecture littérale ne peut guère assurer l'unité entre ces séquences, à moins de faire de l'agent «Grelot * l'acteur syncrétique de fonctions successives:

- faire rentrer la Trotteuse dans le rang;

- assurer le regroupement des vaches;

- évoquer une joie de l'enfance;

- faire retrouver la même joie.

La simplicité de la lecture * dénotative » se fait au détriment de la richesse du texte. En l'occurrence, nous pouvvons exploiter notre analyse précédente et interpréter la première séquence comme l'exemple d'une victoire de la Loi sur le Désir et la deuxième, comme l'exemple inverse d'un plaisir pris maigré 
(ou en dehors de) la loi. La paronomase relevée (trotteuse-déserteurs) grêce a une interprótation opérationnelle (l'intertextualite) permet de voir dens le titre une métephore au plan rhétorique et une condensation dans une perspective psychanalvique.

Nous dirons enfin un mot d'un autre sens indirect d'échelon propositionnel: la tentative de clessement des etres selon des criteres onthropomorphiques: les vagabonds, les distraits, les curieux, les rêveurs... II s'agit à l'évidence d'une typologie.

\section{*UN MOEILE.}

Marcel a trouvé une farmille de marguerites - une vingtaine - groupées d longs intervalles sur une meme souple tige. De ces ordinaires marguerites des champs, blanches, au cour jaune clair. Ce qui en faisait la beauté c'était leur eparpillement gracieux rattaché pourtant au méme naud. II les a placées dans un vase goulot etroit sur une table basse d'où elles debordent dans presque toute le pièce. Elles sont si délibes, si délicates qu'un bref courant d'air les fait toutes vibrer.

- Un mobile de fleurs l ai-je félicite mon mari.

Mouffette ma petite chatte, a sauté sur la table. Ello a tapó une flour comme alle aurait frappe une note de clavier; toutes les fleurs ont fremi. Ello en a atteint une autrè un peu plus haut; méme resultat. Alors Moulfette a tourné la tête avec un air de dire:

- Quel beau jouetl

- Moulfette, non. laissel avons-nous commence par protester. De tout l'été nous ne trouverons peut-être pareil clavier de marguerites.

Elle, une patte en l'air, s'amusait follement. C'était si gracieux ce jeu du petit chat noir et blanc jonglant avec les fleurs frémissantes... nous avons fini par te laisser faire.

je mets celle marguerite en branle; dix-neuf autres marguerites vibrent. Je táche d'arréter le brelan; tout repart de plus belle.

Mouffette, suspendue aux douces marguerites, la patte sur lune. Ia patte sur lautre, a l'air d'un petit sonneur de cloche.

Le NT félicite Marcel (tel est le prénom du mari de Gabrielle Roy) pour avoir découvert une famille de marguerites toutes raltachées a la même.tige et dans laquelle il voit un mobite, puis un instrument de type clavecin lorsque la chatte Mouflette met en branle d'un coup de patte l'ensemble des fleurs. Le NT, d'abord inquiet, puis admiratif, finit par prendre une part active au jell.

On essiste a une double translormation:

- de l'objet découvert (les fleurs) en élément de décoration, puis en instrument de musique: 
- de l'animal de décoration (la chatte) en un agent: pianiste, puis sonneur de cloches;

- du sujet connaissant et amateur (le NT) en spectateur, puis en participant a un jeu ou à un programme musical.

Que peut apporter à cette lecture qui touche déjà à l'interprétation. l'étude des sens indirects?

En fait l'incursion dans l'intransitif est bien modeste. Dire que le bouquet découvert est un mobile de fleurs est effectuer une comparaison plutôt qu'une métaphore puisque les deux termes sont rapprochés * in presentia». Il en est de mème du clavier de marguerites.

Nous ne nous attarderons pas non plus sur les comparaisons introduites par «comme , ni par les copules $\alpha$ air de dire , \&a l'air dont les fonctions ont été mises à jour précédemment. Nous remarquerons toutefois l'usage du conditionnel dans a elle aurait frappé * qui redouble l'effet d'irréel ou d'hypothèse déjà suggéré par «comme». La répétition fréquente de cet indice de sens indirect nous engage à considérer ce métaplasme comme une tentative timide de suggérer la perméabilité entre deux univers, le réel et de l"imaginaire. Telle est du moins l'hypothèse que nous formulons et qu'une étude thématique devrait confirmer. Mais nous pourrions aller plus loin et anticiper la lecture globale de Cet été qui chantait. Dans une perspective extratextuelle, nous dirons alors que le parcours moyen symbolise (entre autres choses) le rêve platonicien d'harmonie universelle.

Revenons à un jeu de relations intratextuelles. On rélève la répétition des verbes a.frémir * et avibrer*. A ces deux formes d'identité totale (frémir et frémir) et à celles d'identité partielle, d'ordre sémantique (frémir et vibrer), s'ajoutent deux similitudes partielles tant sur le plan phonique que sémantique (atapper* et afrapper $*$ ). Enfin pour couronner cette incursion dans le symbolisme lexical, on trouve une permutation d'unités graphiques élémentaires dans «brelan $\approx$ et $\propto$ branle s. Cet écart fait probleme.

Est-ce une paronomase, c'est-à-dire un effet recherché par le NT?

Est-ce un effet de parallélisme inconscient, l'expression (mais où est-elle? dans «branle*? ou dans «brelan»?) influençant le signifié (mais où est-il?)?

Est-ce une coquille? (ce qui ferait du typographe un producteur de sens).

L'étude de ce symbolisme lexical va nous aider à exploiter un symbolisme propositionnel contenu dans l'avant-dernier paragraphe dont la rigueur de construction est remarquable. Le voici:

- une action: mise en branle; conséquence: vibration du tout;

- une action contraire: arrêter; conséquence : vibration du tout.

L'impuissance du NT est manifestée par l'expression verbale x tâcher dex. équivalent à l'aspect $\propto$ non accompli» et surtout par l'expression hyperbolique 
* de plus belle.. Ce remerquable parallélisme symaxique be joint a une autre flgure, l'ellipse des articulations de cause b effet.

Une telle condensation met en relief la double implication de l'actualisation d'une action et de l'impossibilité subséquente de son annulation.

Comme il est souhaitable que l'interprétation se situe à un niveau raisonnable d'abstraction, nous dirons que l'acte positif consiste animer un ensemble d'êtres par contrôle de l'un d'eux. Par contre, la cessation de I'animation n'est pes de la compétence du NT. Ce qui, en définitive, est impliqué dans ce paragraphe est que la vie existe a l'étet potentiel dans le nature, qu un - heureux * haserd ou qu'une touche megique permet parfols de la réveler mais que l'apprenti-sorcier n'a pes le pouvoir de l'arréter, pas plus que celui de la crear.

Le recours a l'intertextuallie va nous permettre une fols de plus de reconnaitre cette implication (équivalent propositionnel de la métonymie) d'une incapacité du NT a contróler le processus de la vie. Nous ne citerons que Rose Anne, victime comme sa mere et bien d'autres d'incessantes grossesses, sa fille Florentine, toutes deux personnages principaux de Bonheur d'occasion;

Plus press de nous, dens Cet ete qui chantair, c'est l'inqulétude délirente des pluviers, pendent la nidification, les 60 petits de la grenoullle, et enfin. en revenant au texte meme, c'est les 19 merguerites obligés de vibrer liunisson pour le plus grand plaisir des spectateurs.

Le paradigme des agents transmetteurs (ou initiateurs) de la vie (le vent, le NT, la chatte) forme l'embryon d'une typologie des magiciens tout comme les distraits, les curieux, amorçaient dans aLa Trotteusen, une typologie des caractères *. La variété de ces agents, leur commutabilité, l'aspect contingent de leurs interventions dans des histoires singulières, tout cela montre les limites de leur pouvoir et implique l'existence d'un *grand horloger *, d'un Destinateur qui délègue sa compétence ou investit provisoirement ses commettants d'une lorme de grace.

A la commutabilité des agents transmetteurs dont le rôle actantiel s'apparente à celui d'adjuvant, correspond le syncrétisme de l'acteur sujet, la lamille de marguerites, et la pluralité de ses lonctions, tour a lour et successivement : objet de décoration, jouet, instrument de musique, cloche pour appeler les lidèles. Par contre, il semble être possible de condenser toutes ces fonctions en une seule. la fonction érotique. L'objet aFamille de marguerites * prend alors une valeur de symbole au sens freudien. Nous ne nous attarderons pas sur les détails évidents qui permettent une telle transposition. Notons que le cadeau, fait par le mari, de cet objet-symbole offre les avantages du plaisir renouvelable, durable, socialement admissible et avouable, mais ne posséde pas certains aspects effrayants de l'objet réel (cf. la procréation incontrôlable). La lamille de marguerites permettant une classification a la fois naturelle et cullurelle étant à la fois objet réel et imaginaire len fait il appartient au symbolique lacanien) ráalise aussi la synthèse entre nature et culture. 


\section{¿LA NUIT DES LUCIOLES*}

La nuit est venue. Depuis longtemps mon ami le merle m'a quittée. II doit dormir d'un profond sommeil dans le creux que je sais, au plus serré de la haie de thuya. Dieu ait en garde sa petite vie éphémère! Les étoiles filantes ont filé. $J$ ai fait un souhait. $J$ 'ai souhaité que les enfants de par icine se lassent jamais d'entendre racontre leur planete Terre. Mème si de nos jours l'on recoit des nouvelles de la Lune!

Après avoir brillé vivement, les étoiles ont pâli. A présent, elles sont a demi effacées comme des clous dédorés au plafond d'une vieille chapelle abandonnée à elle-même. Et je sais tout à coup pourquoi les étoiles s'effacent à demi. C'est pour laisser aux lucioles leur tour de briller. Les voici apparaître par centaines dans la nuit tiede et tranquille.

Il reste tout juste un rien de musique oresque imperceptible suspendue d la cime des arbres. On les dirait frémissant dans leur rêve. Je ne peux me résigner à rentrer. Certaines nuits tres rares, il semble que ce serait crime de ne pas veiller encore un peu avec elles. Non cette fois, parce que chargées de l'angoisse du monde, mais au contraire parce que pénétrées de la plus mystérieuse joie. Et voici donc apparaître les porteuses de flamme.

Tout d'abord, au ras du sol, sur la pelouse fraichement tondue, elles brillent a brefs éclats tout comme le phare dont les jeux intermittents signalent aux bateaux le passage de la pointe de rlle-aux-Coudres.

S'éteignent, se rallument sans trêve sur l'herbe noire d'innombrables phares minuscules comme pour guider dans la nuit d'invisibles voyageurs. $C e$ pourrait être vous, ce pourrait être moi, qui avons souvent à chercher notre chemin.

Puis les humbles petites creatures montent dans les airs avec leur feu. et les voila danseuses de ballet. Et virevoltel Et tourne sur soi! Et pivote, le diadème au front! Le ciel en est tout plein. II est impossible de les suivre toutes a la fois du regard. Qui donc a inventé cette chorégraphie d'une inépuisable fantaisie? Feu là-haut, feu plus loin encore, et feu tout à coup presque sous ma mainl Je l'aurais eu plus vive que jaurais pu saisir cette flamme volante. Berthe m'a raconté en avoir plus d"une fois capturé quand elle était enfant. Pour ma part, je craindrais, par maladresse, de briser le mécanisme délicat qui fait jaillir la courte flamme bleue. Ce qui m'inquiète toutefois, c'est de penser que, de jour, je pourrais très bièn me tromper et prendre pour un insecte banal l'une de ces petites célébrantes du feu.

Pourquoi existent-elles? On dit qu'elles annoncent du temps chaud, mais sans doute annoncent-elles beaucoup plus.

Les voici un peu calmées. Au lieu de danser dans les airs, elles sont devenues de simples promeneuses. A mi-chemin entre ciel et terre, elles passent et repassent avec leur petite lampe suspendue dont le feu nous est révélé ou caché selon les détours de la mystérieuse promenade. 
La nuit est d'une douceur indicible. On pourrait se croire au seuil de 'rinfini, préts a toucher enfin au but vers lequel tend notre espoir, inconnu de nous. Les flammes courtes continuent de voltiger dans te noir brouille.

Leur existence est fugitive. Peut-Atre les lucioles no vivent-elles que le temps de briller un instant d'un vif éclat.

\section{Comme nous tous d'eilleurs I}

Heureux ceux qui, du moins avant de s'éteindrè, auront donné leur plein sclat!

\section{Pris au fou de Dieul}

"La nuit des lucioles* raconte le remplacement de la forme d'une manlfestation de la vie, le scintllement des étolles, par une autre forme, plus proche de nous, mleux observable, les erabesques d'un insecte de nult, le fuclole. Si l'éclosion des lucioles est cyclique, le spectacle offert eu NT est singulier en raison de son intensité.. de sa perfection jusqu'ici inégalée. C'est donc l'émergence du degrés exceptionnel de la qualité d'un phénomène naturel qui lait l'événement et qui constitue par là même le texte en récit.

La nuit apparait comme la mise en suspension de la vie représentée par le sommeil du merle, fait d'ordinaire angaissent (cf, paragraphe 3: inon cette fois parce que chargées de l'angoisse du monde, mals parce que...m). Aussi, le NT cherche-t-il à combler ce vide apparent, ce silence des espaces infinis, ou plutôt, a le taire combler en apostrophant le créateur dans une formule objurgatoire que signale l'emploi du mode subjonctif («Dieu ait en garde... i) de la locution verbale descriptive "avoir en garden.

L'énoncé de la formule objurgatoire peut se traduire de façon plus abstraite: protection contre ce qui est éphémère et fragile (la vie) par l'annulation d'un phénomène naturel (la nuit) effrayant parce qu'il rappelle que l'homme est mortel. Quant l'étude de l'énonciation, elle nous permet d'observer la distribution des rôles. II est clair que le NT est l'allocuteur et qu'il sert d'intermédiaire (pythie 7 prêtre ? magicien? simple fidèle?). Le bénéliciaire est le merle et par synecdoque généralisante, toutes les créatures de la terre. Quant à l'allocutaire, Dieu, il semble avoir une double fonction : agent de l'action de préservation et objet du discours (référent, délocutaire).

Le fait que l'allocuteur (le NT) a l'intention de provoquer une autre action par la parole et le fait que celui auquel il s'adresse est absent permet de classer le discours analysé comme magique. A l'intérieur de ce cadre, le lait que l'acteur auquel le NT s'adresse est le méme que celui qui doit accomplir l'action classe la formule dans la sous-espèce des discours religieux et plus spécifiquement de la priere.

Ainsi est affirmée l'existence d'un univers eutre que celui où se manifestent les phénomènes naturels, univers déjà suggéré par les figures recensées, en particulier les comparaisons. Ce qui est ajouté ici n'est pas seulement la ressemblance entre les êtres qui peuplent ces univers. mais aussi l'orientation des relations hiérarchiques de leurs lieux d'habitation. 
Le NT suggère donc que la connaissance du cosmos est possibte gràce à une intervention de type discursif, la prière ou le charme. La réponse est alors imaginée selon une triple logique de la métonymie, de la métaphore et de l'implication. Les êtres appartenant à des séries différentes (hommes, insectes, plantes...) sorit regroupés parce qu'ils sont en position de contiguïté dans l'univers connu au sein d'un ensemble plus grand, le cosmos. L'identité de fonction (éclat, beauté) des habitants de chacun des deux univers (étoiles. lucioles) postulée autorise leur commutation (procédé métaphorique). L'identité des habitants ainsi décrétée impliquo enfin l'identité de leurs lieux d'habitation.

L'acte perturbateur inconnu est ici la pâleur subite des étoiles (réelle ou imaginée, peu importe). Ce phénomène physique de dimension cosmique, explicable par exemple par le passage dune couche nuageuse, est mis sur-le-champ en relation de finalité avec un autre phénomène par hasard contigu, l'éclosion de lucioles. Une telle démarche traduit l'existence chez le NT d'une pensée ordonnatrice sans cesse en éveil qui ne peut supporter ni le désordre, ni le vide. En mème temps, le présent texte confirme le NT dans son statut de fidèle ayant l'oreille du démiurge.

La formule de souhaits qui suit immédiatement la prière atténue l'aspect incantatoire que l'on trouve dans celle-ci. La puissance virtuelle de l'acte de parole en est réduite et, de ce fait, celle de l'énonciateur ci-devant magicien. Par contre, le rôle de l'adjuvant « Dieu » est effacé: le NT reste le seul médiateur tandis que l'agent dont il est parlé est La Terre, et le bénéficiaire, cles enfants de par ici . On peut parler de conjuration pour qu'apparaisse un objet positif: la terre, métonymie de la Beauté de la Terre.

Or, ce référent qui n'était pas là (ou plus là) ne manque pas d'apparaîtreà point nommé sous la forme du ballet des lucioles. En un sens, cette-formule de souhaits s'éloigne de la prière. Le résultat escompté se produisant, elle se révèle être un charme dans la mesure où l'accent est mis plus sur l'objectif, faire apparaitre l'objectif bénéfique, que sur l'invocation comme tel était le cas précédemment.

Dès lors, placées par magie en position d'objet unique de Beauté. les lucioles se doivent d'être représentatives (symboliques) de divers objets de beauté déjà recensées. Elles deviennent le symbolisant au deuxième degré (propriété particulière du système symbolique) d'une série de symbolisants disparates plus ou moins stéréotypés; les étoiles, les phares, les danseuses. les feux-follets, les vestales, les oracles, les promeneuses, les porte-flambeaux et le dernier mais non le moindre, "l'éclat". la luminosité symbolisant euphémisé du désir.

Ainsi, les phénomènes percus comme inexplicables, étranges ou merveilleux sont rabattus, en dernier ressort, dans le domaine de l'intelligible. En fait, leur existence est mise en cause par une énonciation qui modalise les verbes descriptifs ou utilise des verbes d'attitude comme s'ils étaient des verbes descriptifs. Et ils le sont en effet par rapport à l'univers intérieur du sujet parlant et racontant puisqu'ils traduisent aussi bien des états d'âme (cf. "Je ne peux 
me résigner à rentrer». p. 173) que des transformations psychiques (du non savoir au savoir comme, par exemple, dans l'énonciation «t je sais tout à coup...., p. 173).

Nous tiendrons pour acquise cette constatation: le discours du NT est orienté moins vers la réalité extérieure que vers la réalité intérieure chaisie comme référence. L'entreprise de connaissance est en définitive celle de soi. Nous dirons donc que le discours du NT est gnoséologique.

\& La Nuit des lucioles* est le quatrieme et dernier texte reproduit dont l'analyse des formes du discours est systématique. Comme nous nous y étions engagé, nous continuerons l'étude de Cet été qui chantait dans la même perspective mais en accélérant notre démarche.

\section{*LA GATTE DE MONSIEUR EMILE.}

Au bon travail et à la bonnie conduite correspondent les bonnes récoltes produites par une bonne terre. Telle est la pensée de Monsieur Emile qui semble ne pouvoir concevoir le Laid et le Beau, le Bon et le Bien en même temps. Ainsi est excommuniée une parcelle de terrain rebelle qu'il entoure d'une clôture. Voilà pour la permanence.

L'apparition d'une Belle plante est relevée comme un premier fait. Pour l'expliquer, le NT l'associe selon un rapport de cause à effet à un autre fait. tout simplement parce que les deux phénomènes sont contigus dans l'espace et qu'ils le deviennent dans le temps par la grâce de son acte de parole. Le deuxième fait érigé en incidence est la mise d'un individu particulièrement rétif au ban de la société des vaches par Monsieur Emile.

Voilà donc les mauvais réunis a jamais pour le pire. Mais c'est le contraire qui se produit: la gatte se métamorphose en splendide jardin botanique l'été, en féérie l'hiver. Hasard? Agent mystérieux? Agent naturel comme le vent ou l'engrais apporté par la vache? Le NT admet toutes ces causes mais choisit par goût l'intervention de l'exilée.

On pourrait voir dans cette histoire le contre-pied de la parabole de l'ivraie, c'est-à-dire une sorte d'allégorie de la mutation des Idées (le Laid se transformant en Beau), mutation camouflée par un déterminisme de circonstance. Mais selon la loi du genre, le texte devrait être couronné par une morale explicite. Tel n'est pas le cas ici où le NT se contente de reprendre la série causale dans l'avant dernier paragraphe (p. 31), glissant $d$ 'ailleurs de la cause finale possible à la cause certaine (la vache).

«La gatte de Monsieur Emile serait alors plutôt un exemple de phénomène merveilleux jaillissant non du néant, non de phénomènes merveilleux en soi, mais de la combinatoire merveilleuse de phénomenes prosaíques. identifiables méme par les non-initiés. On voit qu'une telle interprétation admirative et naive devant un extraordinaire ou un étrange que l'on a soi-même inventé donne une importance capitale à l'aagenceur», à l'ordonnateur. 
Nous proposons dès lors d'interpréter les soins d'Émile à sa terre comme une conjuration (*que le bon grain soitm) et l'établissement de la clôture comme une commination ( Que l'ivraie disparaisse*). Acte de parole implicite du fidèle auquel répond l'acte de parole du démiurge (le NT) récompensant le Bon cultivateur. (cf. a... Monsieur Émile, qui était soigneux,...* p. 31).

Mais l'histoire de la gatte implique également une sorte de revanche de l'être défavorisé sur ceux qui ont décrété son déclassement. L'apparence est en effet trompeuse pour qui manque d'intuition ou de sensibilité. Monsieur Emile évoque Aimé et. par delá ce rapprochement intratextuel, la famille du NT dans Rue Deschambault (rapport de type intertextuen).

Comme la gatte. Alicia, sceur du NT, est considérée par sa famille comme un objet de honte. Nous citons: ... on tachait de la cacher quand il venait de la visite... (Alicia in Rue Descambauk, p. 130).

La métamorphose de la gatte n'efface-t-elle pas l'absence de miracle dans l'état de santé d'Alicia? N'est-elle pas une condamnation du rejet par la famille de l'enfant déshérité? Réparation d'une injustice de Dieu?, des hommes?, ou expression d'un remords? Ou crainte d'une punition? $N^{\prime}$ 'est-il pas dit: *Heureux les simples en esprit... Justicier. magicien, moraliste, démiurge, sujet aparlant* son fantasme: Le NT semble être tout cela à la fois:... et plus encore.

\section{"JEANNOT LA CORNEILLE.}

* Jeannot la corneille est nettement un drame de l'insensibilité, de l'intransigeance des hommes et de leur incapacité à distinguer les bons êtres et les mauvais. Jeannot est classé «Bon» par le NT parce que:

- rêveur comme lui (43):

- victime d'une brute (blessure ancienne):

- ressemblant aux humains (45):

- habitant l'arbre préféré du NT, le cerisier:

- et surtout prenant plaisir à se balancer comme lui (49).

Le motif de la balançoire est récurrent dans Cet été qui chantait (cf. p. 39 49-106-162-163-165). On le retrouve également dans d'autres ceuvres (cf. le hamac de Rue Deschambault, p. 74). Le recours à l'intertextualité montre que le mouvement pendulaire est pour le NT un puissant introducteur à l'irréel.

Il en est de mème du vent semblable à l'eau qui s'écoule, béni parce que né dans un pays ou règne l'harmonie selon un rapport métonymique entre le géniteur (le pays d'origine idéalisé) et son fils (le vent), tous deux contigus dans un cosmos édénique. Relevons dans ce même registre thématique la métaphore filée de la corneille qui, sur le cerisier perchée, anaviguait de part en part du ciel * (50), «... voyage(ait) ensemble (avec le NT) sur la même vague du temps* (51). Bel exemple d'une condensation, non simplement d'un 
symbolisme lexical mais d'un représentant de transformation, axe actuel de développement des études freudiennes.

Le drame semble donc se dérouler selon les différentes épisodes d'une allégorie du Méchant (le tueur de la corneille), dénoncé, trainé aux gémonies, tandis que la victime est honorée. Mais, là encore, la morale étant plus suggérée qu'explicitée. le terme d'allégorie ne convient pas. Nous lui préfèrerions l'appellation de discours tantastique puisqu'il y a présentation d'événements pour le moins étranges appartenant à l'irréel. Mais, en définitive, on nous prévient qu'ils sont imaginaires. Nous citons: "... on reprenait pied dans ce quion appelle le "réel s et il paraissait insuffisant, étroit, intolérable. (50)n.

Cependant, la dénonciation du code (cf. «ce qu'on appelfe") remet en question l'existence des deux univers. Le signifié du signifiant "réel „ serait en définitive le même que celui du signifiant irréel». Mais la tentative d'annulation de la dichotomie avouée avorte à l'instant même où elle naît comme le montre l'usage de la modalité du paraitre (cl. « il paraissait étroit ...»). Car l'appréhension directe de l'intelligible est fautive pour toute pensée platonicienne.

En fin de compte. il faut entendre que, si deux univers il y a, ils sont caractérisés par leurs signifiés différents, signifiés assimilés à deux données sensibles: l'euphorie et la dysphorie. On peut alors suggérer le signifiant qui conviendrait à chacun d'eux: éden et géhenne.

Bref, le discours royen s'enlise dans les méandres d'une énonciation qui n'ose pas assumer pleinement ses énoncés. Nous rattacherions volontiers cette-manifestation linguistique a une certaine désorganisation de la vie psychique, à un blocage de type *double entrave».

\section{"AMES EN PEINE"}

"Ames en peinen est l'illustration (synecdoque généralisante à l'échelle propositionnelle) de la bi-tonalité de la vie de tous les êtres: «peur et bonheur, effroi et confiance (76). Lè texte est autant I'histoire d'un couple de pluviers anxieux que celle de la transformation d'une flaque d'eau banale en un objet de beauté grâce. à des agents divers: plantes, insectes, batraciens.

Nous profitons de ce texte pour faire remarquer la vision atomisante du NT. Manilestement lobjet de sa connaissance est délimité par son champ visuel. Seigneur et maitre de sa paroisse, en bonne approche cartésienne. il "découpe la difficulté en autant de... micro-univers dont il répertorie les êtres animés ou inanimés. Dès lors, l'espace physique qui circonscrit telle espece ou qui regroupe deux ou trois especces devient lui-même un être. Tel est le cas de la "gatten, des mares ou llaques, du jardin du NT, de l'enclos aux vaches... Le micro-univers est le symbole (le reflétant) d'un ailleurs dont le symbolisé est la somme des qualités (humaines) prêtées aux êtrés qui le peuplent: le Beau, le Laid... 
Il revient donc au NT de découvrir ces îlots, de les décrire, de les décloisonner, mais pour les structurer dans un ensemble plus grand, à la dimension du cosmos.

\section{«LA GRANDE-MINOUNE-MAIGRE»}

«La Grande-Minoune-Maigre » est une illustration par le contraire de la sédentarité des chats, tout d'abord sous la forme résumée et impersonnelle d'une histoire racontée par Aimé. puis sous la forme d'une longue histoire vécue et directement rapportée. Tout se passe comme si l'histoire vécue était si extraordinaire, invraisemblable, qu'il fallait l'appuyer par une autre. Nous nous arrètons ici pour souligner le désir du NT de dire vrai et d'ètre cru.

En effet, la plupart des histoires mettent en jeu un ou plusieurs témoins des événements qu'elles rapportent: les voisines, les amies, les visiteuses et surtout les voisins immédiats Aimé et Berthe. Ainsi, le discours de Cet été qui chantait tend à se démarquer de l'irréel et affiche l'importance de sa fonction dénotative, référentielle, justement parce que le phénomène đécrit est présenté comme extraordinaire. II l'est bien en fait aux yeux du NT qui pense dire vrai: celui-ci cherche simplement à convaincre le lecteur qu'il est un témoin privilégié et, par voie de conséquence et de façon plus inconsciente, qu'il est un interprète hors pair, une sorte d'Hermès.

La crainte de ne pas avoir la confiance du lecteur entraine le NT à multiplier les exemples, à rechercher l'extraordinaire dans l'excès, dans l'intensité du phénomène plutôt que dans sa nature. Si, comme nous le pensons, la recherche de l'extraordinaire conduit aux reproductions figurées du mème fantasme originaire, il n'est pas étonnant de retrouver dans la plupart des textes le même schéma narratif.

A la peur de ne pas être cru s'ajoute celle, complémentaire, de ne pas être vrai. En effet. le NT a conscience qu'il interprète des signes qu'il recoit d'un ailleurs. Mais on peut imaginer qu'à un certain palier, disons le préconscient, il est pris de scrupules quant à la qualité de son interprétation, en particulier en ce qui concerne sa valeur généralisatrice. D'où cette énonciation précautionneuse si souvent signalée que nous examinerons un peu plus loin dans une perspective étiologique.

Dans "La Grande-Minoune-Maigre * l'extraordinaire naît des efforts hyperboliques de la chatte pour suivre les promeneuses. Ils préparent et mettent en relief l'exploit de l'animal, jusqu'à ce jour humilié, qui consiste à trotter sur un rail (cf. «Mais c'est une merveille...*, p. 100). Dès lors, la Grande-Minoune-Maigre se métamorphose de *traine-la-patte», de souffredouleur en athlète olympique.

Le rapprochement avec des textes tels que a La gatte de Monsieur Émile * et, par delà, «Alicia» de Rue Deschambaut, s'impose. A nouveau, un être disgracié dépasse la condition qui lui est faite, étonne son entourage et acquiert une dignité supérieure à celle de ses juges. A nouveau. la leçon implicite est qu'il ne faut pas se fier aux apparences. Toutefois, chaque texte est unique et 
garde sa propre tonalité. Par exemple, "La Grande-Minoune-Maigre " a quelque chose de diabolique, ne serait-ce que dans cette auto-accélération du plaisir d'être admirée (cf. « Sans doute était-ce moins le fait d'avoir couru sur le rail que de nous avoir épatées qui la grisait. Car elle n'en finissait plus de prouesses. o) (p. 101). Si l'on rappelle que les deux promeneuses s'étaient essayées en vain de marcher sur des rails avec la conscience de se conduire comme des petites filles, on conviendra que le NT puisse fantasmer ici l'un de ses trauma.

D'une part, en effet, la transformation. c'est-a-dire la transgression fantasmée, est annoncée (cf. *Quoiqu'il en soit. cette routinière un jour finit par faire ce qu'elle n'avait encore jamais fait et par là a nous épater pour toujours. $\gg$ p. 88). D'autre part, revient l'expression de l'existence d'un montant de jouissance alloué à chaque individu avec la possibilité pour ce dernier de choisir entre un plaisir permis et un autre défendu. Tout au moins c'est l'exploitation que l'on peut faire des réflexions contigües de Berthe et du NT. A propos de la source perdue, Berthe s'écrie: «Maintenant on a le réfrigérateur. C'est mille fois plus commode, mais on a perdu le plaisir de la source.» p. 93). et le NT ajoute un peu plus loin: aC'est à se demander... si ce que l'on gagne à vivre vaut ce que lion perd. $\times$ p. 94).

\section{LES FRERES ARBRES}

La solidarité extraordinaire d'arbres «jumeaux» n'est reconnue par personne sauf par les corneilles qui viennent discourir et se balancer dans leurs branches (106), ce qui est une façon d'attribuer aux oiseaux un bon jugement. Les corneilles sont a leur tour ignorées par les hommes sauł par Wilbrod I'Innocent. L'Implication d'un cloisonnement inadmissible entre les espèces et les individus d'une mème espèce (entre les *innocents * et les soit-disant sains d'esprit par exemple) est évidente.

\section{LA PAIRE}

Ici est à nouveau illustrée la nécessité d'une fraternité qui s'étendrait à tous les êtres mais qui trouverait cependant son meilleur accomplissement entre individus de la même espèce.

\section{LA FËTE DES VACHES}

La réapparition des vaches, cet animal prosaïque, témoigne du fait que le NT ne cherche pas a faire poétique mais qu'il poursuit sérieusement son entreprise de reclassement permanent des êtres qui l'environnent. Un phénomène naturel, un vent $\alpha$ fou o est considéré comme le producteur unique de deux événements: la fête des vaches (telle est l'interprétation du NT) et le désastre du jardin de fleurs. Le texte se termine par la propre réflexion des animaux sur leur droit au bonheur.

L'implication est triple. D'une part, la douleur et le bonheur, pré-existant a l'individu, quantifiables, identifiables, présents simultanément et partout 
en quantités égales ne sont donc pas autre chose que les deux visages opposés du mème ètre, disons, la vie sentimentale.

D'autre part, le texte postule que la répartition de ces quantités complémentaires devrait se faire de façon équitable entre individus. Enfin, cette répartition ne devrait pas être attributive mais distributive, c'est-à-dire se faire par alternance. A la répugnance à penser le contraire simultanément s'ajoute ici l'idée toute janséniste de souffrances inévitables.

\section{DANSE MOUFFETTE}

Des circonstances favorables, la veillée aux chandelles, le jeu des ombres, l'imagination surexcitée du NT et de ses amies, concourent a transformer la chatte Mouffette en animal fabuleux, en démon chargé de faire peur aux humains. Telle est l'interprétation que fait le NT des attitudes de la chatte dont l'aspect spectaculaire culmine dans la répétition incessante de bonds fantastiques.

La réserve du NT et de ses amies se lit dans la *lueur amusée de (leurs) regards humains *. Mais en réalité, il y a fascination réciproque suscitant une transe spirite. un délire hallucinatoire comme en témoignent les imprécations ou comminations des spectatrices (cf. la répétition de l'ordre de danser).

Il faut retenir de ce texte les jeux d'occultation auxquels se livre la chatte, tantôt animal familier, quasi-humain, tantôt monstre. L'alternance de ces deux états, déroute le lecteur prêt à accepter le merveilleux.

\section{LA MESSE AUX HIRONDELLES}

L'église est la mare (la gatte, l'arche de Noé) où se manifeste la solidarité entre les espèces qui peuplent le micro-univers du NT, solidarité fondée sur l'appartenance commune aux Id́ées, qui transcendent toute taxinomie. Le Bien se révèle sur le visage des fidèles qui viennent d'assister à la messe.

\section{«LE JOUR OUं MARTINE DESCENDIT AU FLEUVE»}

C'est l'histoire du retour aux sources d'une cousine de Berthe. A la suite de son déplacement au bord immédiat du fleuve, elle meurt, tout comme la vieille chienne qui l'avait accompagnée. La présentation de l'histoire révèle la sensibilité de la vieille dame aux phénomènes des marées.

II ne serait peut-être pas exagéré de retrouver là le motif d'occultation d'un objet (ou celui de la balançoirè). La marée montante serait le représentant du mouvement vers la présence totale (ou vers l'apex du désir) et le reflux celui d'un mouvement de retrait vers l'absence totale (la mort ou la chute du désir). Alternance présence/absence. désir/mort, mais aussi oscillation parallèle de la pensée soumise à une double entrave: foi/scepticisme: connaissance/ ignorance; rêve/action; réel/imaginaire. 
A l'appui d'une telle paradigmatisation des lantasmes, nous remarquerons que lion rencontre le même mouvement pendulaire dans les formes du discours royen qui passe, sans cesse de l'hyperbole a la litote ou à l'euphémisme, de la répétition à l'ellipse ou au silence, pour ne citer que quelques figures opposées.

\section{"LES VISITEURS DE LA JOURNEE.}

Le NT est émerveillé par divers spectacles, sonores surtout, que des êtres lointains comme l'émetteur de la cantate de Bach, ou plus rapprochés, comme toute une variêté d'oiseaux, lui font parvenir. Il classe chacun d'eux selon une échelle de talents humains; il y a le librettiste, le ténor, le Brummel. l'instrumentiste de renom, l'étoile de ballet, etc. Par contre-pied, les oiseaux servent de référence au comportement $d^{\prime}$ a amis humains " venus lui rendre visite: il y a les pies (jacasseuses), le pinson chanteur (populaire), le colibri (superficiel, léger).

Le jardin du NT est donc un lieu de rencontre privilégié, un centre d'écoute, d'audition et de parade où s'éprouve la vie.

Mais il est un ilot fixe et son habitant dépend du bon vouloir du Grand Emetteur 1) à envoyer des messagers, 2) à les envoyer dans la bonne direction. La poursuite du colibri à travers les massils est/'exemple de la situation précaire du NT. Le messager peut être si petit ou si capricieux que sa présence échappe aux yeux de l'observateur-catalogueur.

C'est donc à la lettre qu'il faut prendre la description du comportement des femmes dans leur poursuite du colibri (cf. a Nous étions comme des folles, courant de ce côté-ci, de ce côté-là...», p. 167), car il s'agit d'un jeu vital. La perte de vue, même momentanée du messager est ressentie commé l'expression du désintéressement du Pouvoir Suprême pour sa créature: il est signe de mort.

Aussi, le onzième visiteur, le merle, est-il le bienvenu puisqu'il prolonge les signes rassurants de la vie dans la phase sinistre de l'alternance jour/nuit, parallèle à celles vie/mort, présence/absence. Dès lors, on comprend mieux le bon tour que le NT joue a l'oiseau en rentrant a la maison acomme si c'était pour de bon. (p. 170). II peut être considéré comme la tentative magique d'intervertir les rôles de récepteur et de messager, de passer du côté des agissants.

En effet, après sa fausse sortie de la scène, le NT retourne à la balancoire ét constate ce qu'il avait prévu sans l'énoncer : all n'y a plus personnen (170). Le résultat escompté, la suppression de la peur de l'absence de signes de vie. est atteint (cl. aje ne me sens plus seule maintenant (170). Par. un tour magique de sa pensée. le NT accéde à une lonction active dans le grand jeu à éclipse de la vie é de la mort. C'est lui le messager qui a rassuré le nouvel hôte qui "dort peut-être déjàn. tout au moins le devient-il pendant un laps de temps suffisant pour que réapparaissent d'autres signes de la présence de vie. Sa réinsertion réassurante en tant qu'élément fini d'un univers fini sera 
immanquablement sanctionnée par les étoiles filantes, signature fulgurante du Démiurge.

Ici encore, se grefferait très facilement une interprétation psychanalytique de la poursuite accélérée de l'oiseau, de sa maîtrise, de sa disparition,... Nous $n$ 'insisterons pas.

\section{«L'ENFANT MORTE»"}

II existe dans la aréalité » un type d'êtres plus têtus, plus rebelles à une classification idéelle que ceux jusqu'ici répertoriés par le NT. Cet être, c'est le cadavre avec tout son poids d'objet que l'on ne peut transcender sans verser ou dans l'absurde si l'on se situe que le plan du discours logique ou dans le délire du discours psychotique, ou dans l'ineffable du discours mystique, ou encore dans l'illusion du discours merveilleux. Nous avons remarqué combien le NT essayait de se démarquer de façon plus ou moins consciente des deux derniers de ces types de discours en utilisant massivement dans son énonciation la modalité de l'incertitude, du doute.

Dans $\propto$ L'enfant mortem, le corps s'impose comme un obstacle; il semble ne pas avoir de place dans le système habituel de classement du NT. Dans un premier temps tout au moins, il ne suggère rien, d'où son embarras. Absence de vie chez l'enfant décédée, réserve et laconisme de ses camarades écoliers. absence des parents, vide de la pièce qui sert de salon funéraire, en voilà assez pour donner à la jeune institutrice fraîchement débarquée dans ce village du bout du monde le sentiment du néant et pour fixer dans sa mémoire un souvenir qui la hantera à l'âge adulte.

Le dilemme, c'est que l'enfant n'est manifestement pas ici, puisqu'elle est morte, tout en y étant, puisque les écoliers sont tristes et veulent parler de leur camarade et que, quasi-morts eux-mêmes, ils négligent la vivante. l'institutrice. La contradiction est finalement levée grâce à une série d'intuitions (de grâces pourrait-on dire) qui permettent la manipulation des écoliers et leur réintégration aux côtés du NT, c'est-à-dire dans la vie. On peut résumer ces interventions de la façon suivante:

- éveil des écoliers en leur proposant une visite à la morte

- éveil plus grand en suggérant de la veiller

- éveil du visage de la morte (vision d'une joie)

- éveil du cadavre entier en le faisant recouvrir de fleurs

- éveil simultané des êtres environnants : plantes...

Tout cela ne se fait pas sans concession au réel (cf. «Tandis que je considérais l'enfant morte, cette expression «pour la vie», comme si on entendait par là une longue existence, me parut la plus téméraire et la plus folle de toutes celles que nous employons à tort et à travers. *) (185). La comparaison est utilisée ici à des fins inverses de son emploi habituel dans le recueil, c'est-à-dire à mettre en doute l'existence de l'imaginaire, du merveilleux. 
En fin de compte, par une asorte d'intuition " (185), le NT arrive à lancer un pont entre les différents éléments présents de la réalité en rabattant le sensible rebelle à toute investigation dans un intelligible déjà reconnu. "L impuissance * (181) à ordonner le monde est effacée. Mais la connaissance de soi n'a pas progressé pour autant depuis l'enfance comme en témoignent les deux interrogations qui clôturent le texte et qui reprennent en écho, presque mot pour mot, l'interrogation initiale. Ces deux fonctions syntaxiques enchassent l'histoire de la jeune morte comme, dans un blason, un écu mis en abyme par un écu plus petit placé en son centre.

\section{,LES ILES,}

Elles constituent la limite de la vision du NT et, par là-même celle de son champ d'observation. L'histoire est jalonnée par:

- le constat de l'existence d'un désir chez le NT f Je n'ai jamais tant désiré aller quelque part que dans ces petites îles, Berthe.\$ p. 196):

- une interrogation sur l'origine de ce désir (cf. a Quel est donc sur notre coeur l'attrait des îles? * p. 198):

- une réponse évasive (aNe serait-ce pas que nous sommes des enfants perdus qui aspirent a un commun rivage ? p. 198).

L'interrogation et la réponse qui la suit sous forme interro-négative constituent les deux dernières phrases du texte. Ainsi, l'entreprise de connaissance achoppe sur l'impossible connaissance de soi ét l'histoire s'achève sur un appel au savoir du lecteur.

L'aveu d'insatisfaction est relié pour la première fois dans le recueil au sentiment d'une faute que l'on étend à tous les êtres par mesure protectrice. Mais le NT n'associe pas le désir de connaître les îles lparadis perdu, ventre maternel, micro-univers plus mystérieux, plus attirant que la "gatte» ou le jardin, plus inaccessible aussi, et surtout plus difficile a identifier parce que composé d'élements indifférenciés) a d'autres manifestations figuratives de sa libido.

\section{*MONSIEUA TOUNG * et}

¿DE RE TOUR A LA MARE DE MONSIEUR TOUNG*.

L'enchassement qui caractérisait aL'Enfant morte" se retrouve ici à l'échelle du recueil. Faisons tout d'abord l'analyse des deux textes.

Monsieur Toung. ouvre le recueil sur une note triste. La disparition a la fin de la premiere histoire, de ce ouaouaron amene, courtois, quoique laconique, laisse présager un drame: il a été probablement gobé par un héron. Son lieu d'habitation, une mare $*$ du bout du monde isolée des dures réalités quotidiennes, découverte et fréquentée de temps à autre par le NT et son amie. r'est donc pas un éden. 
De retour a la mare de Monsieur Toung traduit le sentiment d'euphorie des mèmes visiteuses alors qu'elles constatent que leur micro-univers n'a jamais été aussi animé. Canards et, ò miracle. pluviers rassurés, cohabitent en harmonie: Tout est donc pour le mieux dans le meilleur des mondes. La lai de l'alternance bonheur-malheur semble vérifiée et le recueil peut paraître se terminer sur une note optimiste: quelque part, un jour, le ouaouaron sera heureux à nouveau.

Mais le ouaouaron n'est quand même pas la... L'imperfection de la création reste flagrante comme le note le NT: aAlors il nous a paru qu'un peu pius loin, dans la paix murmurante des lieux, les oiseaux nous reprochaient nos pauvres questions humaines et nous rappelaient * Tous ne sont pas heureux au mème moment... Un jour c'est l'un, le lendernain l'autre. Quelques-uns jamais, hélas... (203).

On ne peut donc pas glisser le symbolisé que l'on veut sous le symbolisant. Et il est significatif que cette vérité soit transmise au NT let le monde n'est pas harmonieux mais l'homme ne le sait pas, ne veut pas ou ne peut pas le voir. La supériorité que le NT se reconnaît est qu'il est à l'écoute permanente des signes et qu'il s'efforce de les décrypter pour le plus grand bénéfice de tous. Tout au moins croit-il à sa générosité.

Sur le plan mythique, il y'a rapport de substitution d'un motif (le ouaouaron) à un autre (canards, pluviers). Mais il y a également rapport de transformation entre les signifiés du premier texte et ceux du dernier: on assiste au retour à la vie, à l'harmonie, à la joie.

Sur le plan gnoséologique, les choses sont plus nuancées. A la formule * il n'est pas là et tout est dépeuplé * succède xil n'est toujours pas là mais la vie continues. Certes, les trois oiseaux tiennent un discours relativement optimiste. Le voici: «lci on est heureux. . Quand on sera heureux ensemble. ce sera le paradis... (204). Mais leur projection dans l'imaginaire temporel et spatial n'est pas prise en compte par le NT. Son énonciation se démarque de l'énoncé.

Notre démarche générale exclut tout exercice académique du genre coda ou conclusion. Cependant, le dernier texte reprend l'histoire du premier sous un titre sensiblement analogue. Un tel macro-chiasme sémantique et formel impose une dernière interprétation à l'échelle du recueil.

Selon le principe du parallélisme entre le plan de l'expression et celui du contenu, nous postulerons l'équivalence de titre comme impliquant une équivalence sémantique. Nous ne cherchons pas ici à généraliser l'application d'un principe discutable de Jakobson mais plutôt à l'éprouver. D'ailleurs. si le lecteur avait quelque répugnance à suivre une démonstration qui s'engage sur un tel terrain, nous l'inciterions à voir dans les deux titres en question une figure bien connue dont l'effet est incontesté: nous voulons parler de la paronomase.

En outre, selon leur position en abyme, les sept textes enchassés devraient refléter les sens des deux textes enchassants. 
Brel, les deux macro-figures se combinent pour produire un effet de nivellement des contenus. Une lecture littérale globale qui viserait le message comme tel, aurait donc tendance à nier l'existence de toute transformation du sens dans le recueil. Les neuf textes formeraient une description poétique des micro-univers qui environnent le NT. It reste a savoir si notre analyse détaillée des sens indirects, par conséquent anti-poétique, s'éloigne d'une telle lecture.

Le symbolisé du premier texte enchassant peut être réduit à aéclipse inquiétante et inattendue d'un signe de viem. Le symbolisé des sept textes intermédiaires peut être présenté ainsi : «dilficulté d'une enquête sur le monde: multiplicité, variété, plurivocité, mobilité des signes; doutes sur l'interprétation proposée ; confusion entre le réel et l'imaginaire. " Enfin, le symbolisé du dernier texte se résumerait de cette façon: "Phase rassurante de l'alternance signe/absence de signes; mais hypotypose prêtée aux interprétés, non prise en compte par l'interprétant *.

La relative équivalence entre symbólisés nous permet de parler de $\mathrm{Cet}$ éłé qui chantait comme d'un discours descriptif plutôt que narratif puisqu'il n'v a pas vraiment de transformation de contenus. La lecture détaillée des sens indirects rejoint donc celle des macro-structures.

Mais notre étude du symbolisme dans Cet été qui chantait s'est appuyée sur l'analyse des sens directs, laquelle impose la lecture référentielle suivante:

- signifié du texte de départ: “drame naturaliste dont le sujet est la précarité de la vie.»

— signifié des textes intermédiaires: « animation plus ou moins merveilleuse et dramatisée des micro-univers qui composent lenvironnement du NT.

- signifié du texte d'arrivée: *Vie, paix et joie retrouvées dans lun des micro-univers. "

On voit que la lecture des sens directs (des signes) met a jour une transformation des contenus. En effet, les textes intermédiaires sont autant de récits de combats incertains entre les êtres, gagnés tantôt par les uns, tantôt par les autres. Le dernier récit présentant une mare régénérée, on peut donc parler du discours narratif ou mythique de Cet ete qui chantait.

Tandis que l'étude descriptive, analytique, des sens indirects (des symboles) a révélé un été qui a plutôt mal chanté.

Mais peut-on laire une lecture poétique soutenue du recueil? Peut-on se satisfaire d'un sens littéral?

1. Nous avons utilisé un ouvrage publié par les Éditions internationales Alain Stanké Ltée, 1979 (collection 10/10). 